\title{
EFEITO DE UMA SESSÃO DE TREINAMENTO DE FORÇA SOBRE A QUALIDADE DO SONO DE ADOLESCENTES
}

\author{
EFFECT OF A SESSION OF STRENGTH TRAINING ON THE QUALITY \\ ADOLESCENTS SLEEPING
}

Artigo Original

ORIGINAL ARTICLE

Artículo ORIGINAL

\begin{abstract}
EFECTO DE UNA SESIÓN DE ENTRENAMIENTO DE FUERZA EN LA CALIDAD DEL SUEÑO DE LOS ADOLESCENTES
\end{abstract}

\begin{abstract}
Ladyodeyse da Cunha Silva Santiago ${ }^{1}$ (Educador Físico) Maria Julia Lyra' (Educador Físico) Moacyr Cunha Filho² (Engenheiro) Pedro Weldes da Silva Cruz ${ }^{3}$ (Educador Físico)

Marcos André Moura dos Santos ${ }^{4}$ (Educador Físico)

Ana Patrícia Siqueira Tavares Falcão ${ }^{5}$ (Educador Físico)
\end{abstract}

1. Universidade de Pernambuco, Programa de Pós-Graduação em Hebiatria (UPE/ESEF), Recife, PE, Brasil.

2. Universidade Federal Recife,

Programa de Pós-Graduação em Biometria e Estatística Aplicada da (UFPRE), Recife, PE, Brasil.

3. Universidade de Pernambuco (UPE), Faculdade Boa Viagem e Centro Universitário Maurício de Nassau, Grupo de Pesquisa Exercício Físico e Doenças Crônicas não Transmissíveis, Recife, PE, Brasil. 4. Universidade de Pernambuco (UPE), Grupo de Pesquisa em Hemodinâmica e Metabolismo do Exercício e Programa de PósGraduação em Hebiatria,

Recife, PE, Brasil.

5. Universidade de Pernambuco

(UPE), Grupo de Pesquisa em

Avaliação da Performance Humana, Recife, PE, Brasil e Grupo de Pesquisa Educação, Saúde e Meio Ambiente, Vitória de Santo Antão (IFPE), PE, Brasil.

\section{Correspondência:}

Marcos André Moura dos Santos Rua Arnóbio Marques, 310. Escola Superior de Educação Física, Santo Amaro. Recife, PE. Brasil. 50100-130. mmoura23@gmail.com

\section{RESUMO}

Introdução: o sono é uma função biológica fundamental para a conservação da energia e a restauração do metabolismo energético. Objetivo: analisar o efeito de uma sessão do treinamento de força realizada em diferentes horários sobre a qualidade do sono de adolescentes e examinar se a relação entre a melhoria da qualidade do sono e o horário da sessão de treino se altera após o ajuste para idade. Métodos: participaram do estudo seis estudantes do sexo masculino moradores internos do IFPE - Campus Vitória de Santo Antão, $P E$, Brasil. Foram realizadas três sessões de treinamento de força em diferentes horários (manhã, tarde e noite), durante 12 semanas. A escala OMINI-RES foi utilizada para percepção do esforço. A qualidade do sono foi avaliada pelo Índice de Qualidade do Sono de Pittsburgh (PSQI). Ainda foram avaliadas variáveis antropométricas (massa corporal, estatura, IMC) e a composição corporal (\% gordura, massa gorda e massa magra). Resultados: foram observadas diferenças entre as sessões de treino realizadas em diferentes horários e a diagnose de qualidade do sono (manhã: $P<0,001$; tarde: $P=0,001$; noite: $P=0,047$ ). Houve correlação entre a sessão de treino realizada pela manhã $(r=0,95)$ e à tarde $(r=0,92)$ e a diagnose de qualidade do sono. Utilizando o modelo de regressão linear, as sessões de treinamento de força realizadas pela manhã $\left(R^{2}=0,91\right)$ e tarde $\left(R^{2}=0,75\right)$ explicaram de forma significativa a melhora da qualidade do sono em adolescentes, mesmo após o controle pela idade. Conclusão: as sessões de treinamento de força realizadas pela manhã e tarde apresentaram melhor resposta de qualidade do sono de adolescentes.

Palavras-chave: exercício físico, distúrbios do sono, adolescência.

\section{ABSTRACT}

Introduction: sleep is a fundamental biological function for energy conservation and restoration of the energy metabolism. Objective: To analyze the effect of a session of strength training performed at different times on the quality of sleep of adolescents and to examine the relationship between improving the quality of sleep and the time of the training session changes after adjusting for age. Methods: participated in this investigation six male students internal residents of IFPE - Vitória de Santo Antão, PE, Brazil. Three strength training sessions were performed at different times (morning, afternoon and evening) for 12 weeks. The OMNI-RES scale was used to perceived exertion. Sleep quality was assessed using the Pittsburgh Sleep Quality Index (PSQI). Anthropometric variables (body weight, height, BMI) and body composition (\%fat, fat mass and lean mass) were evaluated. Results: differences were observed between training sessions at different times and the diagnosis of sleep quality (morning: $P<0.001$; afternoon: $P=0.001$; evening: $P=0.047)$. There was a correlation between the training session held in the morning $(r=0.95)$ and in the afternoon ( $r=0.92)$ and the diagnosis of sleep quality. Using the linear regression model, the strength training sessions held in the morning $\left(R^{2}=0.91\right)$ and afternoon $\left(R^{2}=0.75\right)$ explained the significantly improved quality of sleep in adolescents, even after adjusting for age. Conclusion: the strength training sessions in the morning and afternoon showed better response in the quality of sleep of adolescents.

Keywords: physical exercise, sleep disorders, adolescence.

\section{RESUMEN}

Introducción: el sueño es una función biológica básica en la conservación de la energía y la restauración del metabolismo energético. Objetivo: analizar el efecto de una sesión de entrenamiento de fuerza realizada en diferentes momentos en la calidad del sueño de los adolescentes y examinar si la relación entre la mejora de la calidad del sueño y el horario de la sesión de entrenamiento cambia después del ajuste para la edad. Métodos: participaron del estudio seis estudiantes varones residentes internos de IFPE - Campus Vitória de Santo Antão, PE, Brasil. Tres sesiones de entrenamiento de fuerza se llevaron a cabo en diferentes momentos (mañana, tarde y noche) durante 12 semanas. La escala OMNI-RES se utilizó para el esfuerzo percibido. La calidad del sueño se evaluó mediante el Índice de Calidad del Sueño de Pittsburgh (PSQI). Las variables antropométricas también fueron evaluadas (peso, talla, índice de masa corporal) y la composición corporal (\% de grasa, la masa grasa y la masa magra). Resultados: se observaron diferencias entre las sesiones de entrenamiento realizadas en diferentes momentos y el 
diagnóstico de la calidad del sueño (mañana: $P<0,001$; tarde: $P=0,001$; noche: $P=0,047$ ). Hubo una correlación entre la sesión de entrenamiento realizada en la mañana $(r=0,95)$ y la tarde $(r=0,92)$ y el diagnóstico de la calidad del sueño. Usando el modelo de la regresión lineal las sesiones del entrenamiento de fuerza llevadas a cabo en la mañana $\left(R^{2}=0,91\right)$ y $\left(R^{2}=0,75\right)$ explicaron de modo significativo la mejora de la calidad del sueño en los adolescentes, incluso después del ajuste por edad. Conclusión: las sesiones de entrenamiento de fuerza en la mañana y por la tarde tenían mejor respuesta de la calidad del sueño de adolescentes.

Palabras clave: ejercicio, trastornos del sueño, adolescente.

\section{INTRODUÇÃO}

Estudos epidemiológicos e clínicos tem mostrado uma associação positiva entre a prática de exercícios físicos e o sono ${ }^{1,2}$. Além disto, o exercício é recomendado pela American Sleep Disorders Association (ASDA) ${ }^{3}$, como uma forma de intervenção não farmacológica para melhorar a qualidade do sono. A possibilidade de um aumento no tempo total de sono pode estar associada aos exercícios físicos, pela necessidade de mais sono para restabelecer a homeostase perturbada pelo exercício.

Este resposta parece ser observada tanto de forma aguda, em que não há adaptação à sua duração, quanto de forma crônica, visto que indivíduos treinados apresentam maior tempo total de sono do que sedentários, mesmo quando fora de treinamento ${ }^{4-7}$. Contudo, o horário em que os exercícios são realizados e a própria aptidão física dos indivíduos são variáveis importantes para as respostas em relação ao padrão de sono.

Os estudos que examinaram a associação entre o horário em que os exercícios foram realizados e a melhora na qualidade do sono parecem controversos e inconclusivos, seja por falta de uma padronização quanto ao horário de realização, ou mesmo quanto pelo tipo de exercício praticado 5,8,9. Além disto, em adolescentes os estudos apontam a existência de uma prevalência de sono insuficiente e de má qualidade do sono ${ }^{10,11}$, que pode estar relacionada a determinados fatores biológicos e maturacionais, além das mudanças sociais e comportamentais ${ }^{12}$.

Neste cenário, há uma lacuna de estudos que investigam a melhoria da qualidade do sono utilizando sessões de treinamento de força em diferentes horários, em um grupo de adolescentes. Deste modo, foram estabelecidos para o presente estudo os seguintes objetivos: a) analisar o efeito de uma sessão de treinamento de força realizada em diferentes horários sobre a qualidade do sono em adolescentes; e b) examinar se a relação entre a melhoria da qualidade do sono e o horário da sessão de treino, se altera após o ajuste para idade.

Nossos resultados incitam uma discussão sobre o horário de realização de uma sessão de treinamento de força sobre a melhoria da qualidade do sono em adolescentes, uma vez que esta forma de exercício realizada no horário da manhã e da tarde demonstrou ser mais eficaz na melhoria da qualidade do sono.

\section{MATERIAIS E MÉTODOS}

A amostra foi composta por escolares moradores internos do Instituto Federal de Educação (IFPE), Campus Vitória de Santo Antão, Pernambuco, Brasil. A coordenação geral de assistência ao estudante (CGPE) do Campus realiza uma divisão dos alunos por quarto, onde são alocados até seis estudantes em cada dependência. Para este estudo, inicialmente, foram recrutados voluntariamente 25 escolares, que responderam ao Questionário do Índice de Qualidade do Sono de Pittsburgh (PSQI). Para a primeira etapa de seleção, os sujeitos deveriam apresentar: queixas de sono não reparador (má qualidade do sono), utilizar ou não algum medicamento para dormir e não estarem envolvidos em programas de exercícios físicos. Após esta primeira etapa 12 sujeitos foram previamente selecionados.
Os critérios de inclusão para participação no estudo foram: não apresentar qualquer lesão muscular ou ortopédica que pudesse comprometer a realização das sessões de intervenção, ter realizado atividade física moderada ou vigorosa nas 12 horas que precederam à avaliação e cumprir $85 \%$ das sessões de treinamento. Nenhum dos sujeitos relatou histórico de problemas musculares ou ortopédicos. Assim, após o cumprimento de todos os critérios estabelecidos, apenas seis sujeitos concluíram o experimento. As características dos sujeitos estão descritos na tabela 1.

Todos os participantes, após receberem esclarecimentos sobre os procedimentos e riscos aos quais seriam submetidos, tiveram o Termo de Consentimento Livre e Esclarecido assinados por seus responsáveis legais. O estudo foi aprovado pelo Comitê de Ética e Pesquisa da Universidade de Pernambuco, Recife, PE, Brasil e seguiu as normas estabelecidas da Comissão Nacional de Ética e Pesquisa (CONEP), protocolo: 89.701.

Trata-se de um estudo de intervenção, de caráter exploratório, o qual foi realizado em um período de doze semanas divididas em três sessões experimentais, com intervalo de quatro semanas entre cada sessão. No dia anterior à realização de cada sessão experimental, os sujeitos foram informados sobre qual o horário e turno (manhã, tarde e noite) do dia seria realizada a série de exercícios. Para que todos os sujeitos realizassem as sessões nas mesmas condições e horários, foi realizado um sorteio aleatório na primeira e segunda sessão do turno inicial da intervenção. Após a realização de cada sessão experimental os participantes foram orientados a responder no dia seguinte, o questionário de avaliação da qualidade do sono para verificar o efeito do exercício após uma noite de sono.

Todos os voluntários permanecem em regime de internato, de segunda a sexta feira, conforme normas da instituição. Durante a semana os sujeitos são acordados por volta das seis horas da manhã, realizam higiene pessoal e se dirigem ao refeitório onde recebem um café da manhã padrão (pães, ovos, queijo, suco, leite e frutas), sem haver interferência do pesquisador quanto à quantidade de alimento consumido. Conforme o horário estabelecido para realização da sessão de treinamento, foram realizadas adequações na rotina das atividades diárias dos sujeitos do estudo. O horário de recolhimento dos internos ocorre às 22h00min (momento em que eram orientados a dormir). Durante a realização do

Tabela 1. Características descritivas da amostra (media \pm desvio-padrão, mínimo e máximo).

\begin{tabular}{c|c|c|c}
\hline Variáveis & Média \pm EP & Mínimo & Máximo \\
\hline Idade (anos) & $16.6 \pm 1.4$ & 15 & 18 \\
\hline $\begin{array}{c}\text { Antropometria e } \\
\text { Composição Corporal }\end{array}$ & & & \\
\hline Peso $(\mathrm{kg})$ & $65.4 \pm 6.9$ & 48.5 & 88.0 \\
\hline Estatura $(\mathrm{cm})$ & $167.4 \pm 2.9$ & 161.0 & 178.5 \\
\hline IMC $\left(\mathrm{kg} / \mathrm{m}^{2}\right)$ & $23.2 \pm 2.3$ & 18.7 & 32.3 \\
\hline Percentual de Gordura $(\%)$ & $10.6 \pm 4.4$ & 3.7 & 28.2 \\
\hline Massa Gorda $(\mathrm{kg})$ & $8.0 \pm 4.2$ & 1.8 & 24.8 \\
\hline Massa Magra $(\mathrm{kg})$ & $57.2 \pm 3.8$ & 46.6 & 67.6 \\
\hline
\end{tabular}


estudo, os voluntários não realizaram nenhum outro tipo de exercício físico, de modo que o impacto da sessão de treinamento pudesse ser avaliado de forma isolada.

A sessão experimental foi composta por oito exercícios (cadeira extensora, cadeira flexora, panturrilha e cadeira abdutora, remada baixa, rosca direta, tríceps pulley e elevação lateral), realizada em três séries de oito repetições, com 60\% 1RM, alternada por segmentos ${ }^{13,14}$ nas semanas 1 a 12 com 1 sessão por semana com duração de 50 a 60 min a sessão de treino foi realizada da seguinte maneira: Alongamentos para todos os segmentos corporais (10 minutos); Parte principal: $3 \times 8$ repetições (intervalo: 60 a 90 segundos). Membros inferiores: cadeira extensora, cadeira flexora, panturrilha e cadeira abdutora, e Membros superiores: remada baixa, rosca direta, tríceps pulley e elevação lateral (45 minutos). A percepção subjetiva de esforço (PSE) foi registrada através da escala OMNI-RES (OMNI - Resistance Exercise Scale). Foi solicitado a cada voluntário que apontasse na escala, imediatamente após o término da série, sua sensação percebida de esforço realizado ${ }^{15}$.

A carga em cada exercício foi prescrita com base no teste de 1RM. O teste foi aplicado para a avaliação da força máxima nos exercícios utilizados no programa experimental ${ }^{16}$. Em cada exercício, o teste foi iniciado com aquecimento (10 repetições), utilizando-se aproximadamente $50 \%$ da carga estimada para a primeira tentativa de 1-RM. Após dois minutos de intervalo, os sujeitos foram orientados a realizar duas repetições com a carga estimada para 1-RM. Caso mais de uma repetição fosse realizada adequadamente ou o sujeito não conseguisse completar uma única repetição, a carga foi ajustada para a segunda tentativa. Para cada exercício foram estipuladas até quatro tentativas para a determinação da carga de 1-RM, com intervalo de três a cinco minutos entre as tentativas. Para minimizar possíveis erros na identificação das cargas, as seguintes estratégias foram adotadas: a) a técnica dos movimentos foi explicada e demonstrada pelos avaliadores antes de cada exercício, b) a técnica do movimento de cada exercício foi monitorada e corrigida quando necessário e c) todos os sujeitos receberam incentivo verbal durante a realização dos testes.

Foram realizadas medidas da massa corporal e estatura. A massa corporal foi obtida através da balança de plataforma (Welmy, Brasil), com carga máxima de 150 kg e escala de precisão de 0,1kg, com os sujeitos descalços e usando roupas leves. Para a medida da estatura foi usado um estadiômetro de madeira com precisão de 0,1 cm. O índice de massa corporal (IMC) foi calculado pelo quociente entre massa corporal $(\mathrm{kg})$ e o quadrado da estatura (m).

Para estimativa da composição corporal, foi utilizado o modelo de dois compartimentos (massa gorda e massa magra) adotando-se as medidas de espessuras de dobras cutâneas, utilizando-se adipômetro cientifico (Lange ${ }^{\circledR}$, Cambridge Scientific Instruments, Cambridge, Maryland), com pressão constante de $10 \mathrm{~g} / \mathrm{mm}^{2}$, precisão de $1 \mathrm{~mm}$. Os pontos de coleta foram o bíceps, tríceps, subescapular e supra-ilíaca e seguiram as recomendações descritas previamente ${ }^{17}$. As medidas foram realizadas em triplicata e adotou-se como valor a mediana. A gordura corporal relativa foi estimada a partir do modelo matemático proposto por Deurenber et al. ${ }^{17}$

\section{Avaliação da qualidade do sono}

Para analise da qualidade do sono, foi utilizado o questionário do Índice de Qualidade do Sono de Pittsburgh (PSQI) ${ }^{18}$, que verifica a qualidade do sono no último mês, fornecendo um índice de gravidade e natureza do distúrbio ${ }^{19}$. O questionário possui 19 questões, agrupadas em sete componentes com distribuição de pesos que variam de zero a três. Estes são classificados quanto às queixas relativas ao sono, sendo: qualidade subjetiva do sono, latência do sono, duração do sono, eficiência habitual do sono, distúrbio do sono, uso de medicamentos para dormir e a disfunção diurna. As pontuações desses sete componentes são somadas para atingir um escore de zero a 21 e quanto maior o score, pior a qualidade do sono. Assim, um score de PSQI maior que cinco revela que o indivíduo apresenta grandes dificuldades em pelo menos dois componentes ou dificuldades moderadas em três ou mais componentes ${ }^{20}$. Logo, pontuações de zero a quatro indicam boa qualidade do sono, de cinco a 10 qualidade ruim e acima de 10 má qualidade do sono. Esse instrumento foi validado na versão em português ${ }^{21}$.

\section{Análise Estatística}

Previamente ao início da análise, a normalidade e a homogeneidade de variância dos dados foram analisados através dos testes de Shapiro-wilk e de Levene, respectivamente. Para verificar o efeito da sessão de treino sobre a diagnose da qualidade do sono, foi utilizado o teste $t$ de Student pareado. Em seguida a correlação momento-produto de Person, foi utilizada para verificar a relação entre a diagnose da qualidade do sono, as sessões de treino (manhã, tarde e noite), e as características antropométricas. Após a verificação da correlação entre as variáveis do estudo, os efeitos da sessão de treino sobre a qualidade do sono foram analisados por meio de um modelo de regressão linear múltipla "stepwise". As análises de regressão não foram realizadas com o objetivo de predição das variáveis dependentes, e sim para verificar a influência das variáveis independentes na explicação da variabilidade de cada variável considerada. Para verificar a consistência interna do PSQI foi utilizado o coeficiente alfa de Cronbach (a). Os dados foram analisados utilizando-se o software SPSS versão 17.0 (SPSS, EUA, 2012). O nível de significância adotado em todas as análises foi de $5 \%$.

\section{RESULTADOS}

O valor de consistência interna (Alpha de Cronbach) entre os sete componentes utilizados no presente estudo foi de 0,75. Ao considerarmos o limite mínimo aceito como satisfatório ser de 0,70, considera-se satisfatório o coeficiente de Cronbach obtido pelo instrumento, na população estudada. Relativamente ao PSQI, foi verificado que $100 \%$ dos sujeitos relataram "qualidade subjetiva do sono" como ruim. No componente "latência do sono" 75\% relataram classificação ruim e 25\% muito ruim. Para os componentes "distúrbios do sono" e "disfunção durante o dia" $75 \%$ responderam qualidade ruim e $25 \%$ boa. As componentes "eficiência inicial do sono" e "uso de medicamentos para dormir" seguiram os critérios de elegibilidade para inclusão no estudo.

A tabela 2 apresenta o escore geral (EG) e a diagnose inicial (DI) dos escolares no PSQI e as respectivas comparações com os diferentes períodos de intervenção (manhã, tarde e noite). Foram encontradas diferenças significativas entre a Dl e todas as sessões de treino realizadas nos diferentes turnos (manhã: $P<0.001$; tarde: $P=0.001$; noite: $P=$ 0.047). Quando observada a frequência em cada turno, verifica-se que a sessão de treino realizada pela manhã demonstrou ser mais efetiva na "Boa Qualidade do Sono" (100\%). Já o treino realizado a tarde contribuiu de forma equilibrada uma vez que foram relatados 50\% para "Boa Qualidade do Sono" e 50\% para "Má Qualidade do Sono", enquanto que o

Tabela 2. Comparação entre diagnose inicial do índice da qualidade do sono (PSQI) e as sessões de treino (manhã, tarde e noite).

\begin{tabular}{|c|c|c|c|c|c|c|c|c|c|}
\hline \multicolumn{4}{|c|}{ Sessão de Intervenção/ turno } & \multicolumn{2}{|c|}{$\begin{array}{c}\text { Manhã } \\
(n=6)\end{array}$} & \multicolumn{2}{|c|}{$\begin{array}{c}\text { Tarde } \\
(n=6)\end{array}$} & \multicolumn{2}{|c|}{$\begin{array}{l}\text { Noite } \\
(n=6)\end{array}$} \\
\hline EG & PSQI & & | & & & & & & \\
\hline 0 a 5 & Boa qualidade do sono & - & - & 6 & $100 \%$ & 3 & $50 \%$ & 1 & $25 \%$ \\
\hline 6 a 10 & Má qualidade do sono & 5 & $75 \%$ & - & - & 3 & $50 \%$ & 5 & $75 \%$ \\
\hline$>10$ & Distúrbios do sono & 1 & $25 \%$ & - & - & - & - & - & - \\
\hline
\end{tabular}


treinamento noturno representou 75\% com "Má Qualidade do Sono" e apenas 25\% com "Boa Qualidade do Sono".

A tabela 3 apresenta as análises de correlação entre as variáveis do estudo. A diagnose da qualidade do sono apresentou correlação com as sessões de treino realizadas no período da manhã e tarde. Porém, não foi observada correlação com sessão de treino realizada à noite, como também com as características antropométricas e da composição corporal.

Quanto aos parâmetros encontrados na análise de regressão linear tendo a diagnose de qualidade do sono como variável independente e as sessões de treino como variáveis dependentes, as sessões de treino realizadas pela manhã e a tarde explicaram de forma significativa a meIhora na qualidade do sono independentes da idade. Isoladamente, as sessões de treino realizadas pela manhã e a tarde explicaram em 91\% $\left(R^{2}=0,91\right)$ e $75 \%\left(R^{2}=0,75\right)$ da melhora na qualidade do sono, respectivamente. Estes valores aumentaram após os ajustes para idade (tabela 4).

Tabela 3. Correlação (correlação de Pearson - coeficiente r) entre a diagnose inicial do sono, as sessões de treino, características antropométricas e os testes neuromusculares.

\begin{tabular}{c|c|c|c|c|c|c}
\hline Variáveis & $\begin{array}{c}\text { Diagnose } \\
\text { Inicial do } \\
\text { Sono }\end{array}$ & Peso $(\mathbf{K g})$ & Estatura & \%G & MG (Kg) & MLG (Kg) \\
\hline $\begin{array}{c}\text { Diagnose inicial } \\
\text { do sono }\end{array}$ & - & 0.402 & -0.671 & 0.793 & 0.787 & -0.126 \\
\hline Sessão de treino & 0.958 & 0.262 & -0.757 & 0.721 & 0.702 & -0.286 \\
manhã & $(0.010)$ & $(0.670)$ & $(0.138)$ & $(0.170)$ & $(0.186)$ & $(0.641)$ \\
\hline Sessão de treino & 0.921 & -0.020 & -0.898 & 0.498 & 0.472 & -0.542 \\
tarde & $(0.026)$ & $(0.975)$ & $(0.039)$ & $(0.393)$ & $(0.422)$ & $(0.345)$ \\
\hline Sessão de treino & -0.303 & -0.936 & -0.455 & -0.777 & -0.800 & -0.809 \\
noite & $(0.621)$ & $(0.019)$ & $(0.442)$ & $(0.122)$ & $(0.104)$ & $(0.097)$ \\
\hline
\end{tabular}

Tabela 4. Coeficientes de regressão linear (estimativas beta; intervalos de confiança de $\% 95$ e P-valor), entre as sessões de treino em jovens e a melhoria na qualidade do sono.

\begin{tabular}{c|c|c|c|c}
\hline \multicolumn{5}{c}{ Diagnose de Qualidade do Sono } \\
\hline & $\boldsymbol{\beta} \pm$ s.e. & $\mathbf{9 5 \%}$ IC & $\boldsymbol{R}^{2}$ & $\boldsymbol{P}$ \\
\hline Sessão de treino manhã & $0,867 \pm 0,149$ & 0,393 a 1,342 & 0,91 & 0,010 \\
\hline Sessão de treino manhã + idade & $1,047 \pm 0,262$ & $-0,082$ a 2,176 & 0,94 & 0,057 \\
\hline Sessão de treino tarde & $0,602 \pm 0,199$ & 0,032 a 1,236 & 0,75 & 0,057 \\
\hline Sessão de treino tarde + idade & $0,994 \pm 0,220$ & 0,048 a 1,940 & 0,92 & 0,046 \\
\hline Sessão de treino noite & $-0,205 \pm 0,372$ & $-1,390$ a 0,980 & 0,92 & 0,692 \\
\hline Sessão de treino noite +idade & $0,631 \pm 0,205$ & $-0,250$ a 1,512 & 0,93 & 0,091 \\
\hline
\end{tabular}

\section{DISCUSSÃO}

No presente estudo, o diagnóstico inicial sobre a qualidade do sono em adolescentes demonstrou que mesmo nesta faixa etária são encontrados casos relacionados a distúrbios do sono. Neste contexto, estudos têm relatado a existência de alta prevalência de sono insuficiente e de má qualidade do sono entre jovens ${ }^{10,11}$. Amostras representativas de estudantes do Ensino Médio do estado de Santa Catarina, Brasil, em 2001 e 2011, identificaram prevalências de má qualidade (26,3\% e 34,5\%) e de duração insuficiente (37\% e 54\%) em estudantes do ensino médio22.

Mais recentemente, foi realizado um estudo para verificar comportamentos associados ao sono entre jovens brasileiros estudantes de 20 escolas públicas do Recife e Florianópolis, foi observado nos dados transversais e longitudinais que 45,7\% e 45,8\% dos jovens relataram má qualidade e $76,7 \%$ e $77,5 \%$ reportaram duração insuficiente do sono, respectivamente. Na análise transversal, a menor prática de atividade física (OR = 0,74; IC 95\%: 0,55; 0,99) e o maior consumo de salgados (OR $=1,67 ;$ IC 95\%: 1,18; 2,36) estiveram associados à qualidade negativa do sono, enquanto o tempo excessivo de televisão (OR=0,48; IC 95\%:
0,30; 0,75) e de consumo de refrigerantes (OR=1,84; IC 95\%: 1,19; 2,84) associaram-se à duração insuficiente do sono ${ }^{23}$

Diante de um quadro que demonstra a existência de diferentes fatores associados à má qualidade de sono em jovens, a adoção de um maior nível de atividade física parecer contribuir na melhoria da qualidade do sono. No presente estudo, foi observado que as sessões de treinamento de força realizadas no período da manhã (100\%) e tarde (50\%) demonstraram ser mais efetivas na melhoria da qualidade do sono. Além disto, mesmo com uma variação na frequência em relação à percepção obervada no PSQI, todas as sessões de treino demonstraram um efeito agudo desta forma de exercício em relação à qualidade do sono em adolescentes.

Neste contexto, os estudos que examinaram a existência de relações ou modificações na qualidade do sono em adolescentes, encontram resultados controversos, seja pelo tipo de exercício ou a intensidade com que são praticados. Contudo, alguns estudos têm descrito associações positivas entre o envolvimento em atividade física e a melhor qualidade e duração do sono ${ }^{24,25}$. Noland et al. ${ }^{8}$ identificaram que a prática de exercícios físicos antes do horário de dormir foi citada como uma das estratégias utilizadas pelos adolescentes para adormecer. Porém, este achado é contrário ao encontrado no presente estudo, uma vez que na sessão de exercícios realizadas à noite apenas 25\% dos sujeitos relataram boa qualidade do sono.

Para além destas observações, vale destacar que a maioria dos estudos, que tem analisado a melhoria na qualidade do sono em adolescentes apresentam delineamentos de caráter puramente descritivo e de associação, não sendo relatados, estudos com delineamento experimental para verificar relações de causa e efeito. Contudo, em adultos o exercício mostrou-se capaz de reduzir a latência para o início do sono ou pelo menos não alterá-la ${ }^{4}$. Em um outro estudo foi observada uma melhora no padrão de sono após a prática de exercício físico em indivíduos (homens: 45 e 65 anos de idade) que apresentavam movimento periódico das pernas (MPP), considerado um tipo de distúrbio do sono. Os voluntários apresentaram menor MPP, porém este efeito não foi decorrente das concentrações de dopamina, verificada após a realização do exercício ${ }^{26}$.

Outro aspecto que deve ser considerado é o fato de alguns estudos utilizarem em seus procedimentos, metodologias distintas tanto em relação aos protocolos utilizados, como a forma contrária de observação, ou seja, o efeito da má qualidade do sono afetando o desempenho físico. Neste contexto, parece existir um consenso do não efeito da privação e ou má qualidade do sono no desempenho aeróbio, é importante ressaltar que, as dificuldades relacionadas a estes distúrbios, provocam um aumento significativo na percepção do esforço ${ }^{27}$, e uma redução do tempo para a exaustão, sendo que esses parâmetros afetam o desempenho aeróbio.

Do mesmo modo, Souissi et al. ${ }^{28}$ observaram que o desempenho anaeróbio parece não ser afetado após 24 horas de vigília sustentada. No entanto, foram observadas modificações após 36 horas sem dormir. Além, disto, tem sido sugerido que a privação de sono não possui efeitos aparentes na força e na potência máxima ${ }^{27}$, na contração isométrica máxima ${ }^{5}$, na fadiga muscular e no trabalho total28. Contudo, apesar destes achados, os reais efeitos da privação de sono no desempenho físico ou fisiológico são conflitantes e/ou inconclusivos. As razões para essas disparidades podem ser explicadas pelos diferentes tipos, frequência, intensidade e duração dos exercícios empregados, assim como por outros parâmetros, entre os quais, o tempo de privação e a idade dos sujeitos experimentais ${ }^{29}$.

O segundo propósito do presente estudo foi verificar qual a contribuição do horário da sessão de treino na explicação da melhoria da qualidade do sono, independente da idade dos sujeitos. Os resultados do presente estudo demonstraram que a sessões de treinamento de força realizadas no turno da manhã e tarde foram mais efetivas na explicação 
da melhoria da qualidade do sono em adolescentes. Estes achados são contrários ao que tem sido descrito sobre o melhor horário de realização do exercício e a melhora da qualidade do sono.

Youngstedt et al. ${ }^{5}$ demonstraram que os exercícios realizados próximos ao horário de dormir podem levar a um aumento na latência para o sono e um aumento no sono de ondas lentas, enquanto exercícios realizados pela manhã não o fazem. O mecanismo subjacente parece está associado ao fato de que o exercício ao aumentar a temperatura corporal, criaria uma condição capaz de facilitar "o disparo" do início do sono, por ativar os processos de dissipação de calor controlados pelo hipotálamo, assim como os mecanismos indutores do sono dessa mesma região ${ }^{4}$. Portanto, a duração total do episódio de sono, assim como a quantidade de sono, aumenta em função do aumento do gasto energético ${ }^{4}$.

Por outro lado, tem sido observado que, exercícios demasiadamente intensos e de longa duração podem levar a um período inadequado de recuperação. Existem evidências de que o excesso de atividades podem alterar parâmetros fisiológicos, e também o padrão de sono ${ }^{28}$. No caso dos exercícios, entretanto, existe uma relação em forma de " $U$ " invertido entre a fadiga induzida pelo exercício e a qualidade do sono ${ }^{28}$.

No entanto, no presente estudo a exposição aos exercícios foi realizada em apenas uma sessão de treinamento de força em diferentes horários, ou seja, sem a observação de efeitos crônicos. Deste modo, é possível que tanto a o tipo do exercício utilizado, com também a intensidade e horário que foi praticado possam apresentar respostas distintas. Um estudo realizado por De Faria et al. ${ }^{2}$ com homens (20 a 40 anos de idade) que apresentavam boa qualidade do sono, realizaram uma sessão de treinamento de força em diferentes horários (manhã:7:00-9:00h; tarde: 13:00-15:00h; e noite: 18:00-20:00h) com uma carga equivalente a 50\% do teste de 1-RM. Foi obervado que indivíduos sedentários com boa qualidade de sono não apresentaram alterações significativas em seus parâmetros de sono após a realização de uma sessão treinamento de força.

Contudo, no presente estudo, a população estuda foi composta por adolescentes que ainda se encontram no periodo da puberdade, onde

\section{REFERÊNCIAS}

1. De Mello MT, Fernandez AC, Tufik S. Levantamento epidemiológico da prática de atividade física na cidade de São Paulo. Rev Bras Med Esporte. 2000;6(4):119-24.

2. de Faria AP, Cavagnolli DA, Rossi MV, Ferreira SE, Bittencourt LRA, Tufik S, et al. Effects of resistance exercise on the sleep patterns of sedentary individuals. Sleep Sci. 2009;2(3):141-6.

3. Thorpy MJ. The international classification of sleep disorders: diagnostic and coding manual. Rochester, Minnesota: ASDA; 1991.

4. Driver HS, Taylor SR. Exercise and sleep. Sleep Med Rev. 2000;4(4):387-402.

5. Youngstedt SD, O'Connor PJ, Crabbe JB, Dishman RK. The influence of acute exercise on sleep following high caffeine intake. Physiol Behav. 2000;68(4):563-70.

6. Chervin RD. Sleepiness, fatigue, tiredness, and lack of energy in obstructive sleep apnea. Chest. 2000;118(2):372-9.

7. Awad KM, Drescher AA, Malhotra A, Quan SF. Effects of exercise and nutritional intake on sleep architecture in adolescents. Sleep Breath. 2013;17(1):117-24.

8. Noland H, Price JH, Dake J, Telljohann SK. Adolescents' sleep behaviors and perceptions of sleep. J Sch Health. 2009;79(5):224-30.

9. Buxton OM, L'Hermite-Balériaux M, Turek FW, van Cauter E. Daytime naps in darkness phase shift the human circadian rhythms of melatonin and thyrotropin secretion. Am J Physiol Regul Integr Comp Physiol. 2000;278(2):R373-82.

10. Cain N, Gradisar M. Electronic media use and sleep in school-aged children and adolescents: a review. Sleep Med. 2010;11(8):735-42.

11. Matricciani L, Olds T, Petkov J. In search of lost sleep: secular trends in the sleep time of school-aged children and adolescents. Sleep Med Rev. 2012;16(3):203-11

12. Carskadon MA, Acebo C, Jenni OG. Regulation of adolescent sleep: implications for behavior. Ann N Y Acad Sci. 2004;1021:276-91.

13. Kraemer WJ, Adams K, Cafarelli E, Dudley GA, Dooly C, Feigenbaum MS, et al. Progression models in resistance training form healthy adults. Med Sci Sports Exerc. 2002;34(2):364-80.

14. Lloyd RS, Faigenbaum AD, Stone MH, Oliver JL, Jeffreys I, Moody JA, et al. Position statement on youth resistance training: the 2014 International Consensus. Br J Sports Med. 2014;48(7):498-505.

15. Robertson RJ, Goss FL, Rutkowski J, Lenz B, Dixon C, Timmer J, et al. Concurrent validation of the OMNI perceived exertion scale for resistance exercise. Med Sci Sports Exerc. 2003;35(2):333-41.

16. Kraemer WJ, Fry A, Ratamess N, French D. Strength testing: development and evaluation of metho- a produção de hormônios relativos ao genero são exarcerbadas, pelo próprio processo da maturidade, além da propria idade, que está diretamente relacionada à ocorrência destes eventos ${ }^{14}$. Além disto, a rotina diária imposta pelo regime de internado exige dos sujeitos do presente estudo o cumprimento de horários pré-estabelecidos, tanto para dormir quanto para acordar, fato que pode ter contribuido em um menor tempo necessário para recupar o organismo da sobrecarga imposta pelo exercicio, principalmente ao ser realizada no periodo da noite. Assim, a natureza do próprio exercício e as condições em que são particados parecem exercer uma influência direta sobre a qualidade do sono, provocando respostas distintas, bem como adaptações fisiológicas correspondentes ${ }^{2}$.

Nesta fase, a importância do sono na recuperação entre as sessões de treinamento torna-se mais evidente, tendo em vista a associação entre a secreção de hormônio de crescimento $(\mathrm{GH})$ e o sono de ondas lentas, podendo-se inferir a importância de um sono adequado para a restauração corporal. Além disto, o perfil noturno de GH mostrou-se útil para indicar alterações nas funções anabólicas normais que ocorrem durante o sono, induzidas pelo exercício realizado durante o dia ${ }^{14}$.

\section{CONCLUSÃO}

Os resultados obtidos neste estudo sugerem relação positiva entre a melhoria da qualidade do sono e as sessões de exercícios realizadas pela manhã e à tarde em adolescentes sedentários; todavia essa relação parece ser diferente após ajustes pela idade dos indivíduos, de forma que maiores coeficientes de correlação são observados. Como a aptidão física parece ser altamente responsiva por uma melhora qualidade do sono, estudos de intervenção com maior período de tempo são necessários para investigar o potencial efeito do exercício na melhora da qualidade do sono em adolescentes.

Todos os autores declararam não haver qualquer potencial conflito de interesses referente a este artigo. dology. Champaign, IL: Human Kinetics; 1995. p.115-38.

17. Deurenberg P, Weststrate JA, Seidell JC. Body mass index as a measure of body fatness: age- and sex-specific prediction formulas. Br J Nutr. 1991;65(2):105-14.

18. Buysse DJ, Reynolds CF 3rd, Monk TH, Berman SR, Kupfer DJ. The Pittsburgh Sleep Quality Index: a new instrument for psychiatric practice and research. Psychiatry Res. 1989;28(2):193-213.

19. Togeiro SM, Smith AK. Diagnostics methods for sleep disorders. Rev Bras Psiquiatr. 2005;27(Suppl 1):8-15.

20. Bertolazi AN. Tradução, adaptação cultural e validação de dois instrumentos de avaliação do sono: Escala de sonolência de Epworth e índice de qualidade de sono de Pittsburgh [dissertação]. Porto Alegre: Universidade Federal do Rio Grande do Sul Faculdade de Medicina; 2008.

21. Chellappa SL, Araujo JF. Qualidade subjetiva do sono em pacientes com transtorno depressivo. Est Psicol. 2007;12(3):269-74

22. Hoefelmann LP, Lopes Ada S, da Silva KS, Moritz P, Nahas MV. Sociodemographic factors associated with sleep quality and sleep duration in adolescents from Santa Catarina, Brazil: what changed between 2001 and 2011? Sleep Med. 2013;14(10):1017-23.

23. Hoefelmann LP, Silva KS, Barbosa Filho VC, Silva JAd, Nahas MV. Behaviors associated to sleep among high school students: cross-sectional and prospective analysis. Rev Bras Cineantropom Desempenho Hum. 2014;16(Suppl 1):68-78.

24. McKnight-Eily LR, Eaton DK, Lowry R, Croft JB, Presley-Cantrell L, Perry GS. Relationships between hours of sleep and health-risk behaviors in US adolescent students. Prev Med. 2011;53(4-5):271-3.

25. Foti KE, Eaton DK, Lowry R, McKnight-Ely LR. Sufficient sleep, physical activity, and sedentary behaviors. Am J Prev Med. 2011;41(6):596-602.

26. Cavagnolli DA, Esteves AM, Castiglione ML, Batista IR, Bressan RA, Tufik S, et al. Dopamine transporter shown by SPECT in patients with periodic leg movement after acute physical exercise. Med Sci Sports Exerc. 2013;45(2):224-9.

27. Montelpare WJ, Plyley MJ, Shephard RJ. Evaluating the influence of sleep deprivation upon circadian rhythms of exercise metabolism. Can J Sport Sci. 1992;17(2):94-7.

28. Souissi N, Sesboüé B, Gauthier A, Larue J, Davenne D. Effects of one night's sleep deprivation on anaerobic performance the following day. Eur J Appl Physiol. 2003;89(3-4):359-66.

29. Antunes HKM, Andersen ML, Tufik S, De Mello MT. Privação de sono e exercício físico; Sleep deprivation and exercise. Rev Bras Med Esporte. 2008;14(1):51-6 